\title{
Concise Synthesis of Arnottin I and (-)-Arnottin II
}

\author{
Fujiko Konno, ${ }^{1}$ Tsutomu Ishikawa, ${ }^{* 1}$ Masatoshi Kawahata, ${ }^{2}$ and Kentaro Yamaguchi ${ }^{2}$ \\ ${ }^{1}$ Graduate School of Pharmaceutical Sciences, Chiba University, \\ Inage, Chiba 263-8522, Japan \\ ${ }^{2}$ Faculty of Pharmaceutical Sciences at Kagawa Campus, Tokushima Bunri University, 1314-1 Shido, \\ Sanuki, Kagawa 769-2193, Japan \\ E-mail: benti@p.chiba-u.ac.jp
}

Table of Contents

General

Preparation of 6-Bromo-2,3-dimethoxybenzaldehyde

Preparation of 6-Bromo-2,3-dimethoxybenzoic Acid

Preparation of Methyl 6-Bromo-2,3-dimethoxybenzoate

Preparation of tert-Butyl 6-Bromo-2,3-dimethoxybenzoate

General Procedure in Table 1

Data of 2-(2-Methylphenyl)-1-tetralone (8, X = 2-Me)

Data of 2-(3,4-Methylenedioxyphenyl)-1-tetralone $\left(\mathbf{8}, \mathrm{X}=3,4-\mathrm{OCH}_{2} \mathrm{O}\right)$

Data of $6 H$-Benzo $[d]$ naphtho[1,2-b]pyran-6-one (9)

Preparation of $( \pm)$-Dihydroarnottin II (( \pm$)-\mathbf{1 2})$

Preparation of (-)-ent-Dihydroarnottin II ((-)-12)

${ }^{1} \mathrm{H}$ NMR of Dihydroarnottin I (3)

${ }^{13} \mathrm{C}$ NMR of Dihydroarnottin I (3)

${ }^{1} \mathrm{H}$ NMR of Arnottin I (1) page

S-3

S-3

S-3

S-3

S-3

S-4

S-4

S-4

S-4

S-4

S-5

S-6

S-7

S-8 
${ }^{13} \mathrm{C}$ NMR of Arnottin I (1) S-9

${ }^{1} \mathrm{H}$ NMR of $(R)-(+)$-Dihydroarnottin II ((+)-12) S-10

${ }^{13} \mathrm{C}$ NMR of $(R)-(+)$-Dihydroarnottin II ((+)-12) S-11

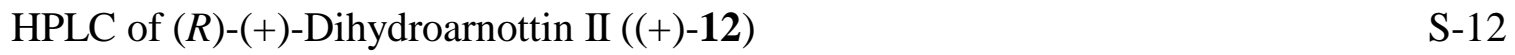

HPLC of $(S)$-(-)-Dihydroarnottin II ((-)-12) S-13

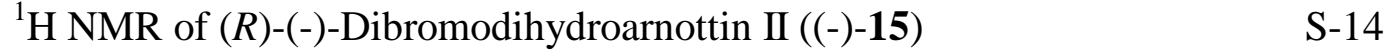

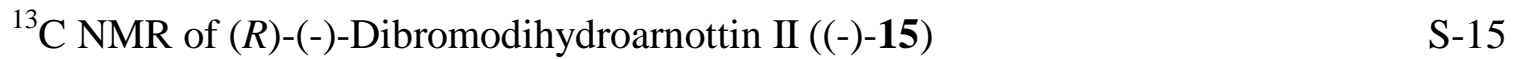

ORTEP Drawing of (R)-(-)-Dibromodihydroarnottin II ((-)-15) S-16

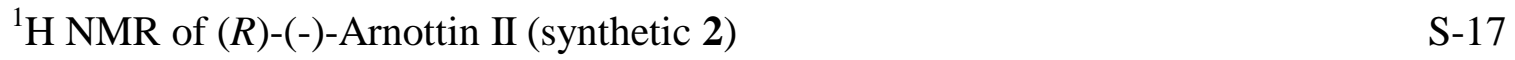

${ }^{13} \mathrm{C}$ NMR of $(R)-(-)$-Arnottin II (synthetic 2) S-18

HPLC of $(R)-(-)$-Arnottin II (synthetic 2) $\quad$ S-19

HPLC of $(R)-(-)$-Arnottin II (natural) $\quad$ S-20

$\begin{array}{ll}\text { References } & \text { S-21 }\end{array}$ 
General: Melting points were uncorrected. For TLC were used $\mathrm{SiO}_{2} 60$ F254 and for column chromatography $\mathrm{SiO}_{2} 60(63-210 \mu \mathrm{m})$.

6-Bromo-2,3-dimethoxybenzaldehyde. The titled compound was prepared from 6-bromo-2-hydroxy3-methoxybenzaldehyde by the reported procedure ${ }^{1}$; mp $82-83{ }^{\circ} \mathrm{C}$ (lit. ${ }^{1} \mathrm{mp} 71-73{ }^{\circ} \mathrm{C}$ ); IR (ATR) $v_{\max }$ $1687 \mathrm{~cm}^{-1} ;{ }^{1} \mathrm{H}$ NMR $\left(400 \mathrm{MHz}, \mathrm{CDCl}_{3}\right): \delta(\mathrm{ppm}) 3.89(\mathrm{~s}, 3 \mathrm{H}), 3.93(\mathrm{~s}, 3 \mathrm{H}), 6.97(\mathrm{~d}, J=8.8 \mathrm{~Hz}$, $1 \mathrm{H}), 7.33(\mathrm{~d}, J=8.8 \mathrm{~Hz}, 1 \mathrm{H}), 10.33(\mathrm{~s}, 1 \mathrm{H}) ;{ }^{13} \mathrm{C} \mathrm{NMR}\left(100 \mathrm{MHz}, \mathrm{CDCl}_{3}\right): \delta(\mathrm{ppm}) 56.1,62.2,112.5$, $117.4,128.4,129.2,152.0,152.6,190.3$.

6-Bromo-2,3-dimethoxybenzoic Acid. The titled compound was prepared by the reported procedure ${ }^{1}$; mp 109-110 ${ }^{\circ} \mathrm{C}$. (lit. ${ }^{1} \mathrm{mp} \mathrm{83-85}{ }^{\circ} \mathrm{C}$ ); IR (ATR) $v_{\max }$ 2941, $1716 \mathrm{~cm}^{-1} ;{ }^{1} \mathrm{H}$ NMR (400 MHz, $\mathrm{CDCl}_{3}$ ): $\delta$ (ppm) 3.88 (s, 3H), 3.93 (s, 3H), 6.87 (d, J=8.9 Hz, 1H), $7.28(\mathrm{~d}, J=8.9 \mathrm{~Hz}, 1 \mathrm{H}), 8.40(\mathrm{br} \mathrm{s}, 1 \mathrm{H}) ;{ }^{13} \mathrm{C}$ NMR (100 MHz, $\left.\mathrm{CDCl}_{3}\right): \delta(\mathrm{ppm})$ 56.1, 61.9, 108.6, 114.9, 128.1, 130.3, 147.0, 152.1, 170.5.

Methyl 6-Bromo-2,3-dimethoxybenzoate. A mixture of 6-bromo-2,3-dimethoxybenzoic acid (2.32 g, $8.89 \mathrm{mmol}), \mathrm{MeI}(31.1 \mathrm{~mL}, 17.8 \mathrm{mmol})$, and $\mathrm{K}_{2} \mathrm{CO}_{3}(21.84 \mathrm{~g}, 13.3 \mathrm{mmol})$ in DMF (15 mL) was stirred at room temperature for $4 \mathrm{~h}$ and poured into $\mathrm{H}_{2} \mathrm{O}(30 \mathrm{~mL})$, and extracted with AcOEt $(10 \mathrm{~mL} \times 3)$. The organic solution was washed with $\mathrm{H}_{2} \mathrm{O}(5 \mathrm{~mL}$ x 2$)$ and brine $(5 \mathrm{~mL})$, dried $\left(\mathrm{Na}_{2} \mathrm{SO}_{4}\right)$, and evaporated. The residue was purified by column chromatography (hexane/AcOEt $=10: 1$ ) to afford the titled compound $(1.94 \mathrm{~g}, 80 \%)$ as a pale yellow oil ${ }^{2}$; IR (ATR) $v_{\max } 1730 \mathrm{~cm}^{-1} ;{ }^{1} \mathrm{H} \mathrm{NMR}\left(400 \mathrm{MHz}, \mathrm{CDCl}_{3}\right)$ : $\delta(\mathrm{ppm}) 3.85(\mathrm{~s}, 3 \mathrm{H}), 3.86(\mathrm{~s}, 3 \mathrm{H}), 3.95(\mathrm{~s}, 3 \mathrm{H}), 6.83(\mathrm{~d}, J=8.8 \mathrm{~Hz}, 1 \mathrm{H}), 7.23(\mathrm{~d}, J=8.8 \mathrm{~Hz}, 1 \mathrm{H}) ;{ }^{13} \mathrm{C}$ NMR (100 MHz, $\left.\mathrm{CDCl}_{3}\right): \delta(\mathrm{ppm})$ 52.6, 56.0, 61.6, 108.7, 114.5, 127.8, 131.2, 146.9, 152.0, 166.3.

tert-Butyl 6-Bromo-2,3-dimethoxybenzoate. Conc. $\mathrm{H}_{2} \mathrm{SO}_{4}(0.33 \mathrm{~mL}, 6.13 \mathrm{mmol})$ was added to a vigorously stirred anhydrous magnesium sulfate $(2.95 \mathrm{~g}, 24.5 \mathrm{mmol})$ in $\mathrm{CH}_{2} \mathrm{Cl}_{2}(30 \mathrm{~mL})$ and the mixture was stirred at room temperature for $15 \mathrm{~min}$. After addition of 6-bromo-2,3-dimethoxybenzoic acid $(1.60 \mathrm{~g}, 6.13 \mathrm{mmol})$ followed by tert-BuOH $(2.9 \mathrm{~mL}, 30.6 \mathrm{mmol})$, the mixture was stirred at room temperature for $18 \mathrm{~h}$, quenched with sat. $\mathrm{NaHCO}_{3}$ aq $(70 \mathrm{~mL})$ and stirred until all magnesium sulfate was dissolved. The organic phase was separated, washed with brine $(5 \mathrm{~mL})$, dried $\left(\mathrm{Na}_{2} \mathrm{SO}_{4}\right)$, and evaporated. The residue was purified by column chromatography (hexane/AcOEt = 9:1) gave the titled compound (1.47 g, 76\%) as a colorless oil; IR (ATR) $v_{\max } 1728 \mathrm{~cm}^{-1} ;{ }^{1} \mathrm{H} \mathrm{NMR} \mathrm{(400} \mathrm{MHz,} \mathrm{CDCl}_{3}$ ): $\delta(\mathrm{ppm}) 1.61(\mathrm{~s}, 9 \mathrm{H}), 3.84(\mathrm{~s}, 3 \mathrm{H}), 3.87(\mathrm{~s}, 3 \mathrm{H}), 6.79(\mathrm{~d}, J=8.8 \mathrm{~Hz}, 1 \mathrm{H}), 7.21(\mathrm{~d}, J=8.8 \mathrm{~Hz}, 1 \mathrm{H}) ;{ }^{13} \mathrm{C}$ $\operatorname{NMR}\left(100 \mathrm{MHz}, \mathrm{CDCl}_{3}\right): \delta$ (ppm) 28.1, 56.1, 61.5, 82.9, 108.7, 114.0, 127.8, 132.7, 146.6, 152.1, 164.7; HREIMS $m / z: 316.0314$ (Calcd for $\mathrm{C}_{13} \mathrm{H}_{17}{ }^{79} \mathrm{BrO}_{4}\left(\mathrm{M}^{+}\right): 316.0310$ ). 
General Procedure in Table 1: Pd-Catalyzed Coupling Reaction of Bromobenzene 6 and 1Tetralone (7). A mixture of bromobenzene 6 (1 mmol), 1-tetralone (7) (1.2 mmol), Pd catalyst (0.01 $\mathrm{mmol})$, ligand $(0.02 \mathrm{mmol})$, and base $\left[\right.$ tert-BuONa $(1.3 \mathrm{mmol})$ or $\left.\mathrm{K}_{3} \mathrm{PO}_{4}(2.3 \mathrm{mmol})\right]$ in $\mathrm{PhMe}(1 \mathrm{~mL})$ was stirred at $80{ }^{\circ} \mathrm{C}$. After dilution with AcOEt $(10 \mathrm{~mL})$, the mixture was filtered through Celite ${ }^{\circledR}$ pat and washed with AcOEt $(10 \mathrm{~mL})$. The combined organic solution was washed with water $(3 \mathrm{~mL})$ and brine $(3 \mathrm{~mL})$, dried $\left(\mathrm{Na}_{2} \mathrm{SO}_{4}\right)$, and evaporated. The residue was purified by column chromatography (hexane/AcOEt $=25: 1$ ) to afford 8 or 9.

2-(2-Methylphenyl)-1-tetralone (8, X = 2-Me). Obtained as colorless needles, mp 63-64 ${ }^{\circ} \mathrm{C}$ (lit. ${ }^{3}$ not reported); IR (ATR) $v_{\max } 1676 \mathrm{~cm}^{-1} ;{ }^{1} \mathrm{H} \mathrm{NMR}\left(400 \mathrm{MHz}, \mathrm{CDCl}_{3}\right): \delta(\mathrm{ppm}) 2.32(\mathrm{~s}, 3 \mathrm{H}), 2.34-2.41(\mathrm{~m}$, $1 \mathrm{H}), 2.43-2.50(\mathrm{~m}, 1 \mathrm{H}), 3.07(\mathrm{ddd}, J=16.7,4.8,4.8 \mathrm{~Hz}, 1 \mathrm{H}), 3.17(\mathrm{ddd}, J=16.7,11.9,4.8 \mathrm{~Hz}, 1 \mathrm{H})$, $3.99(\mathrm{dd}, J=11.9,4.8 \mathrm{~Hz}, 1 \mathrm{H}), 7.05-7.08(\mathrm{~m}, 1 \mathrm{H}), 7.15-7.23(\mathrm{~m}, 3 \mathrm{H}), 7.31$ (d, J = 7.9 Hz, 1H), 7.35 (t, $J=7.5 \mathrm{~Hz}, 1 \mathrm{H}), 7.52(\mathrm{t}, J=7.5 \mathrm{~Hz}, 1 \mathrm{H}), 8.10(\mathrm{~d}, J=7.9 \mathrm{~Hz}, 1 \mathrm{H}) ;{ }^{13} \mathrm{C} \mathrm{NMR}\left(100 \mathrm{MHz}, \mathrm{CDCl}_{3}\right)$ : $\delta(\mathrm{ppm}) 19.9,29.4,30.4,51.5,126.2,126.8,126.9,127.6,127.8,128.8,130.6,133.1,133.4,136.5$, 138.6, 144.1, 198.1; HREIMS m/z: 236.1196 (Calcd for $\left.\mathrm{C}_{17} \mathrm{H}_{16} \mathrm{O}\left(\mathrm{M}^{+}\right): 236.1201\right)$.

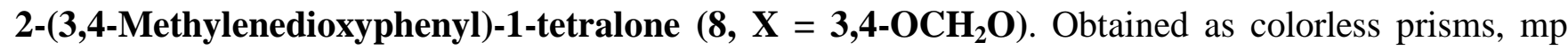
132-133 ${ }^{\circ} \mathrm{C}$ (lit. ${ }^{4} \mathrm{mp}$ 135-136.5 ${ }^{\circ} \mathrm{C}$ ); IR (ATR) $v_{\max } 1674 \mathrm{~cm}^{-1} ;{ }^{1} \mathrm{H}$ NMR (400 MHz, $\left.\mathrm{CDCl}_{3}\right): \delta(\mathrm{ppm})$ 2.34-2.41 (m, 2H), 3.01-3.16 (m, 2H), $3.72(\mathrm{dd}, J=8.1,8.1 \mathrm{~Hz}, 1 \mathrm{H}), 5.94(\mathrm{~s}, 2 \mathrm{H}), 6.65(\mathrm{~d}, J=7.6 \mathrm{~Hz}$, $1 \mathrm{H}), 6.67(\mathrm{~s}, 1 \mathrm{H}), 6.78(\mathrm{t}, J=7.9 \mathrm{~Hz}, 1 \mathrm{H}), 7.28(\mathrm{t}, J=7.9 \mathrm{~Hz}, 1 \mathrm{H}), 7.33(\mathrm{t}, J=7.6 \mathrm{~Hz}, 1 \mathrm{H}), 7.50(\mathrm{t}, J=$ $7.6 \mathrm{~Hz}, 1 \mathrm{H}), 8.08(\mathrm{~d}, J=7.6 \mathrm{~Hz}, 1 \mathrm{H}) ;{ }^{13} \mathrm{C} \mathrm{NMR}\left(100 \mathrm{MHz}, \mathrm{CDCl}_{3}\right): \delta(\mathrm{ppm}) 28.9,31.3,54.1,100.9$, 108.3, 108.8, 121.6, 126.8, 127.8, 128.7, 132.8, 133.4, 133.5, 144.0, 146.5, 147.7, 198.2; HREIMS $m / z: 266.0938$ (Calcd for $\left.\mathrm{C}_{17} \mathrm{H}_{14} \mathrm{O}_{3}\left(\mathrm{M}^{+}\right): 266.0943\right)$.

6H-Benzo[d]naphtho[1,2-b]pyran-6-one (9). Obtained as colorless needles, mp 181-182 ${ }^{\circ} \mathrm{C}$ (lit. ${ }^{5}$ not reported); IR (ATR) $v_{\max } 1712 \mathrm{~cm}^{-1} ;{ }^{1} \mathrm{H}$ NMR (400 MHz, $\mathrm{CDCl}_{3}$ ): $\delta$ (ppm) 2.91-2.95 (m, $2 \mathrm{H}$ ), 3.03 (dif $\mathrm{t}, J=7.9 \mathrm{~Hz}, 2 \mathrm{H}), 7.22-7.33(\mathrm{~m}, 3 \mathrm{H}), 7.49(\mathrm{dd}, J=7.9,7.3 \mathrm{~Hz}, 1 \mathrm{H}), 7.61(\mathrm{~d}, J=8.1 \mathrm{~Hz}, 1 \mathrm{H}), 7.76(\mathrm{dd}$, $J=7.3,7.1 \mathrm{~Hz}, 1 \mathrm{H}), 7.87(\operatorname{dif} \mathrm{d}, J=7.9 \mathrm{~Hz}, 1 \mathrm{H}), 8.35(\mathrm{~d}, J=7.9 \mathrm{~Hz}, 1 \mathrm{H}) ;{ }^{13} \mathrm{C} \mathrm{NMR}(100 \mathrm{MHz}$, $\left.\mathrm{CDCl}_{3}\right): \delta(\mathrm{ppm}) 21.0,27.2,109.5,120.9,122.1,122.8,127.0,127.5,127.6,128.6,129.2,130.2$, 134.7, 136.3, 137.3, 148.1, 162.1; Anal. Calcd for $\mathrm{C}_{17} \mathrm{H}_{12} \mathrm{O}_{2}$ : C, 82.24; H, 4.87. Found: C, 82.19; H, 4.90 .

( \pm )-6,7-Methylenedioxy-1-tetralone-2-spiro-3'-(6,7-dimethoxyphthalide) (( \pm -Dihydroarnottin II) 
(( \pm )-12). To a solution of $3(18 \mathrm{mg}, 0.05 \mathrm{mmol})$ in $\mathrm{CH}_{3} \mathrm{CN}(4 \mathrm{~mL})$ were added a $4 \times 10^{-4} \mathrm{M}$ solution EDTANa $\mathrm{E}_{2} \cdot 2 \mathrm{H}_{2} \mathrm{O}(0.08 \mathrm{mmol})$ in $\mathrm{H}_{2} \mathrm{O}$ and 1,1,1-trifluoroacetone $(0.45 \mathrm{~mL}, 5.0 \mathrm{mmol})$ with vigorous stirring at $0{ }^{\circ} \mathrm{C}$ and then a pulverized mixture of oxone $(307 \mathrm{mg}, 0.50 \mathrm{mmol})$ and $\mathrm{NaHCO}_{3}(126 \mathrm{mg}$, $1.50 \mathrm{mmol}$ ) with a small portion. The mixture was stirred at $0{ }^{\circ} \mathrm{C}$ for $0.5 \mathrm{~h}$ and then $2 \mathrm{~h}$ at room temperature, diluted with water $(5 \mathrm{~mL})$, and extracted with $\mathrm{CHCl}_{3}(3 \mathrm{~mL}$ x 3$)$. The combined extracts were washed with brine $(5 \mathrm{~mL})$, dried $\left(\mathrm{Na}_{2} \mathrm{SO}_{4}\right)$, and evaporated. Purification of the residue by column chromatography (benzene/acetone $=100: 3)$ followed by recrystallization from AcOEt gave $( \pm)$-12 $(9$ $\mathrm{mg}, 47 \%$ ) as colorless prisms, mp $183-184{ }^{\circ} \mathrm{C}$ (lit. $\left.{ }^{6} \mathrm{mp} 186-187^{\circ} \mathrm{C}\right)$.

\section{(S)-(-)-6,7-Methylenedioxy-1-tetralone-2-spiro-3'-(6,7-dimethoxyphthalide) (ent-Dihydroarnottin}

II) ((-)-12). Treatment of 3 with $\mathrm{AD}-\mathrm{mix}-\beta$ in place of $\mathrm{AD}-\mathrm{mix}-\alpha$ under the same conditions described in the preparation of dihydroarnottin II ((+)-12) in Experimental section for $95 \mathrm{~h}$ afforded entdihydroarnottin II ((-)-12), mp $145-147{ }^{\circ} \mathrm{C}$, as colorless needles in $85 \%$ yield; Anal. Calcd. for $\mathrm{C}_{20} \mathrm{H}_{16} \mathrm{O}_{7}$ : C, 65.22; H, 4.38. Found: C, 65.13; H, 4.38; HRFABMS m/z: 369.0984 (Calcd for $\mathrm{C}_{20} \mathrm{H}_{17} \mathrm{O}_{7}$ $\left.\left(\mathrm{M}^{+}+\mathrm{H}\right): 369.0974\right) ;[\alpha]_{\mathrm{D}}^{24} 63\left(c 0.1, \mathrm{CHCl}_{3}\right) ; 88 \%$ ee by chiral HPLC (CHIRALPAK IA, $0.46 \mathrm{~cm} \mathrm{x}$ $25 \mathrm{~cm}) ; n$-hexane $/ \mathrm{CH}_{2} \mathrm{Cl}_{2} / \mathrm{EtOH}=15: 5: 4$; flow rate $=0.5 \mathrm{~mL} / \mathrm{min}$; detection wavelength $=254 \mathrm{~nm} ; t_{\mathrm{R}}$ $(\operatorname{minor})=14.4 \mathrm{~min}, t_{\mathrm{R}}($ major $)=21.8 \mathrm{~min}$. 


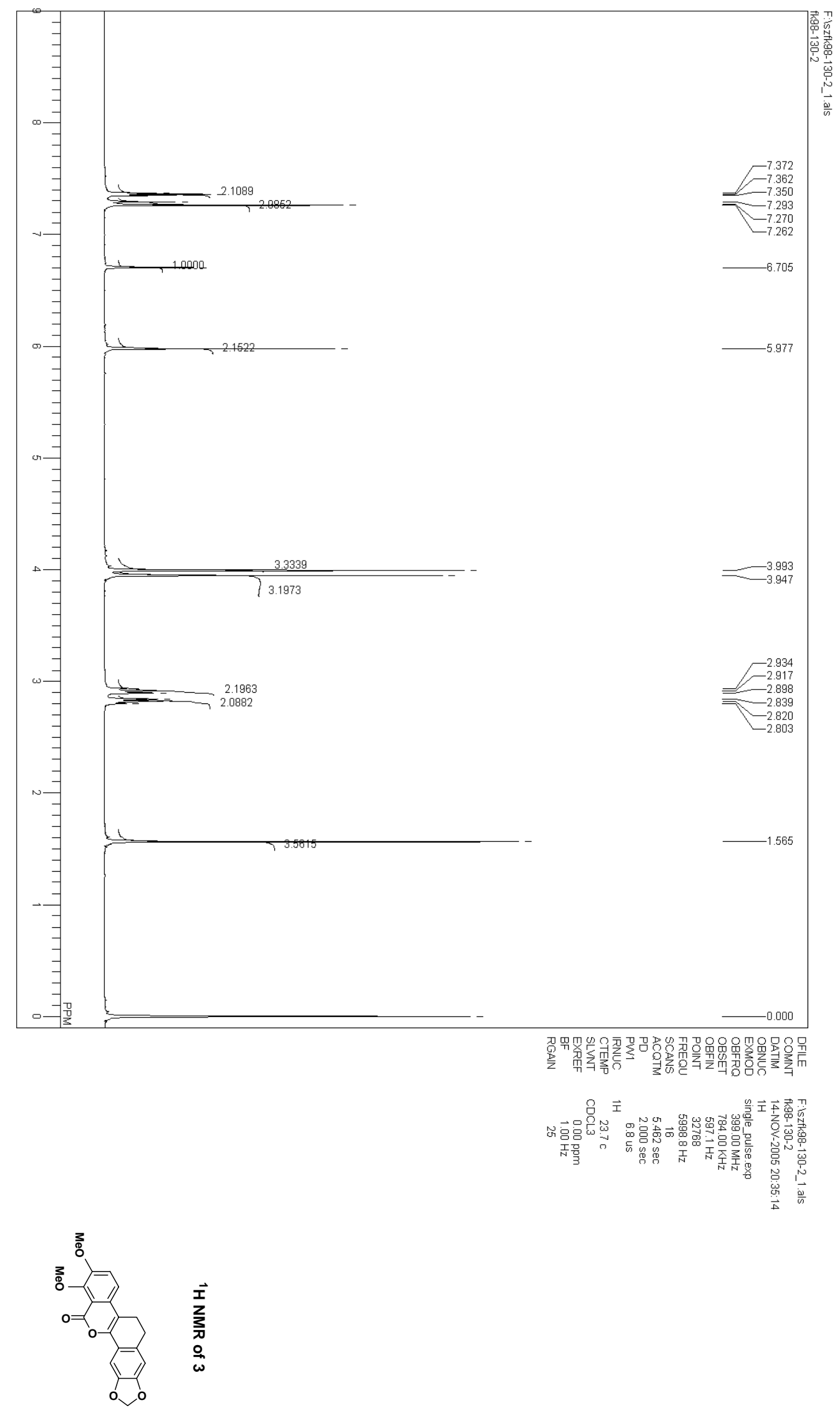



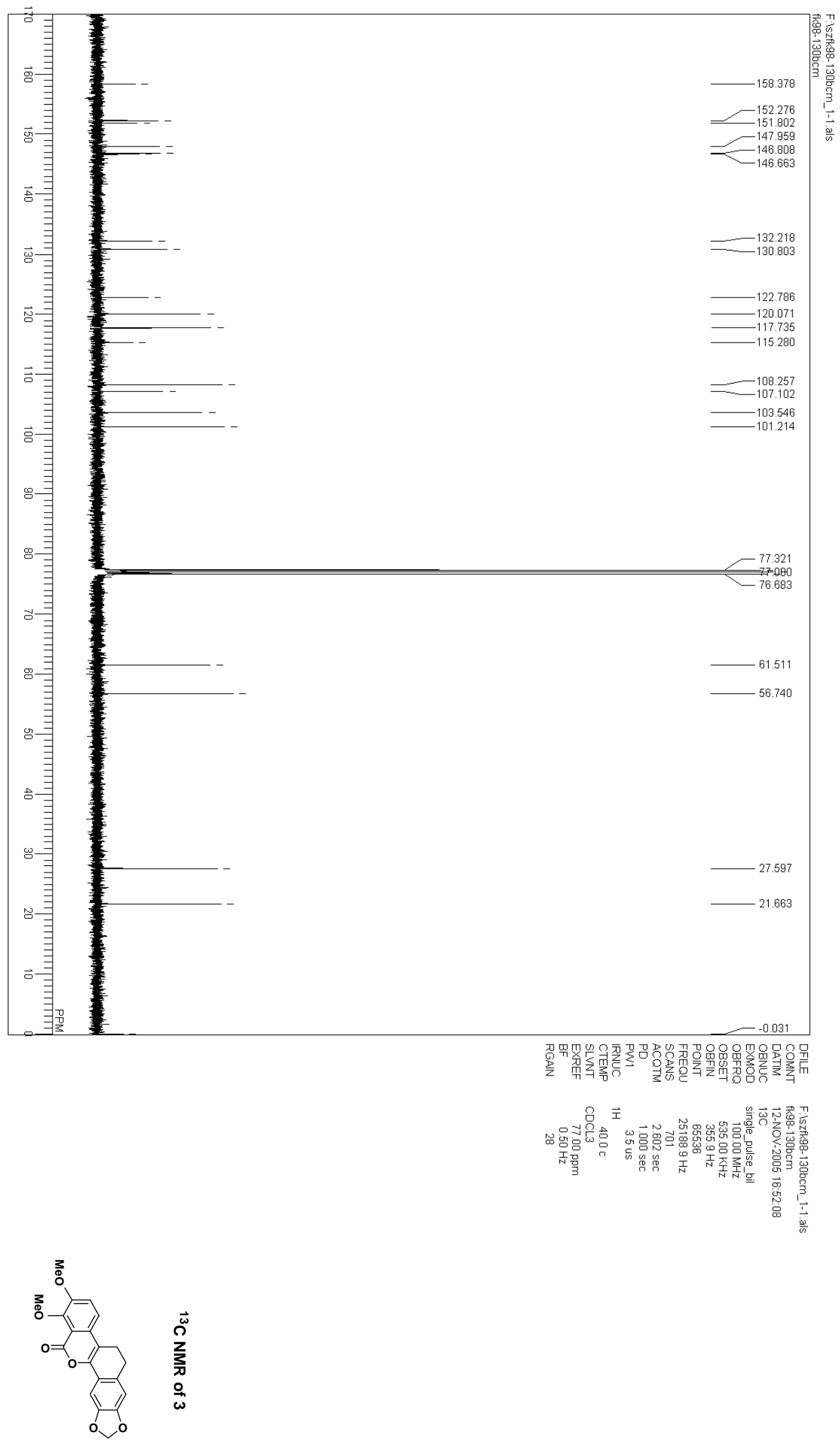

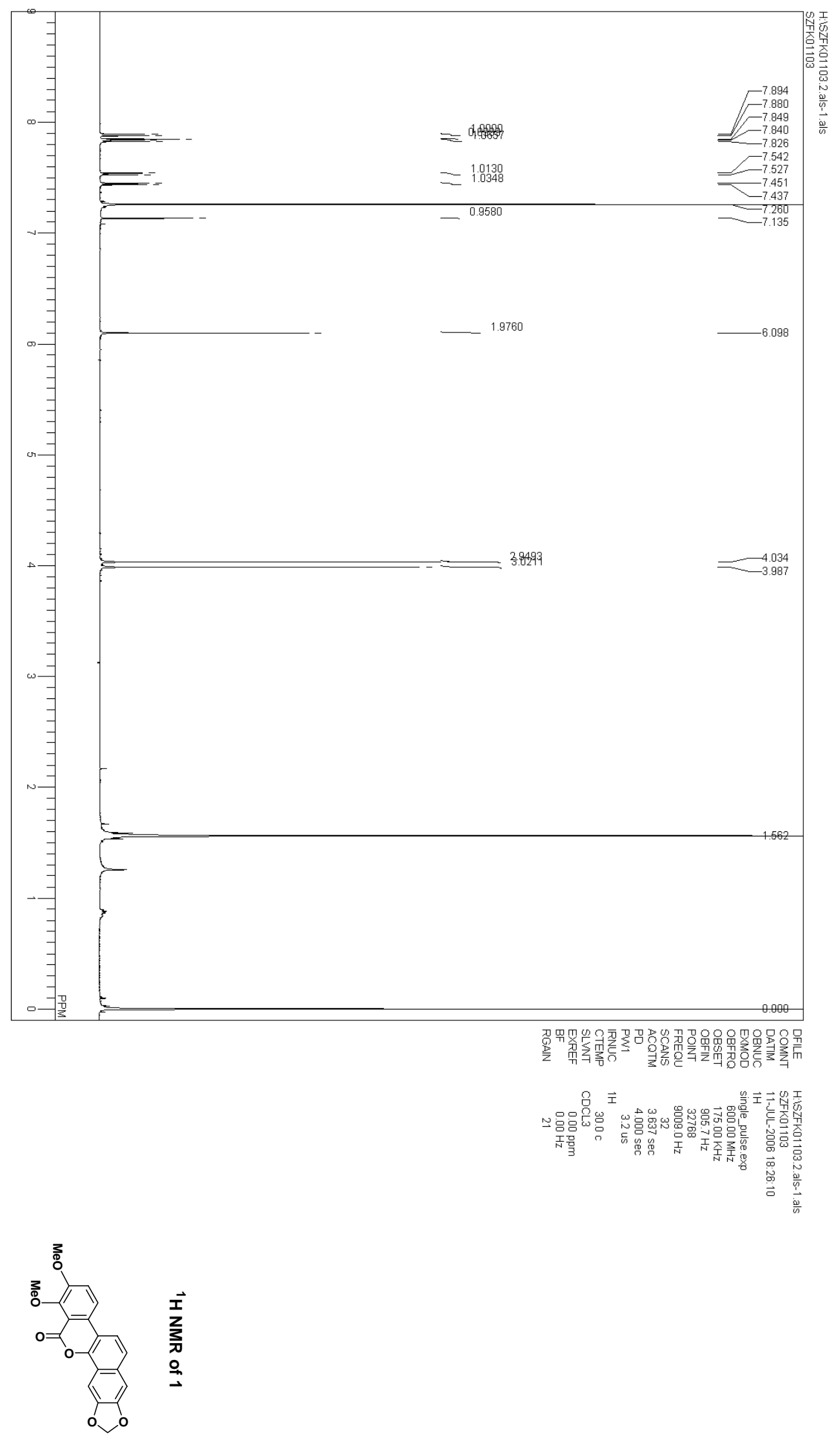


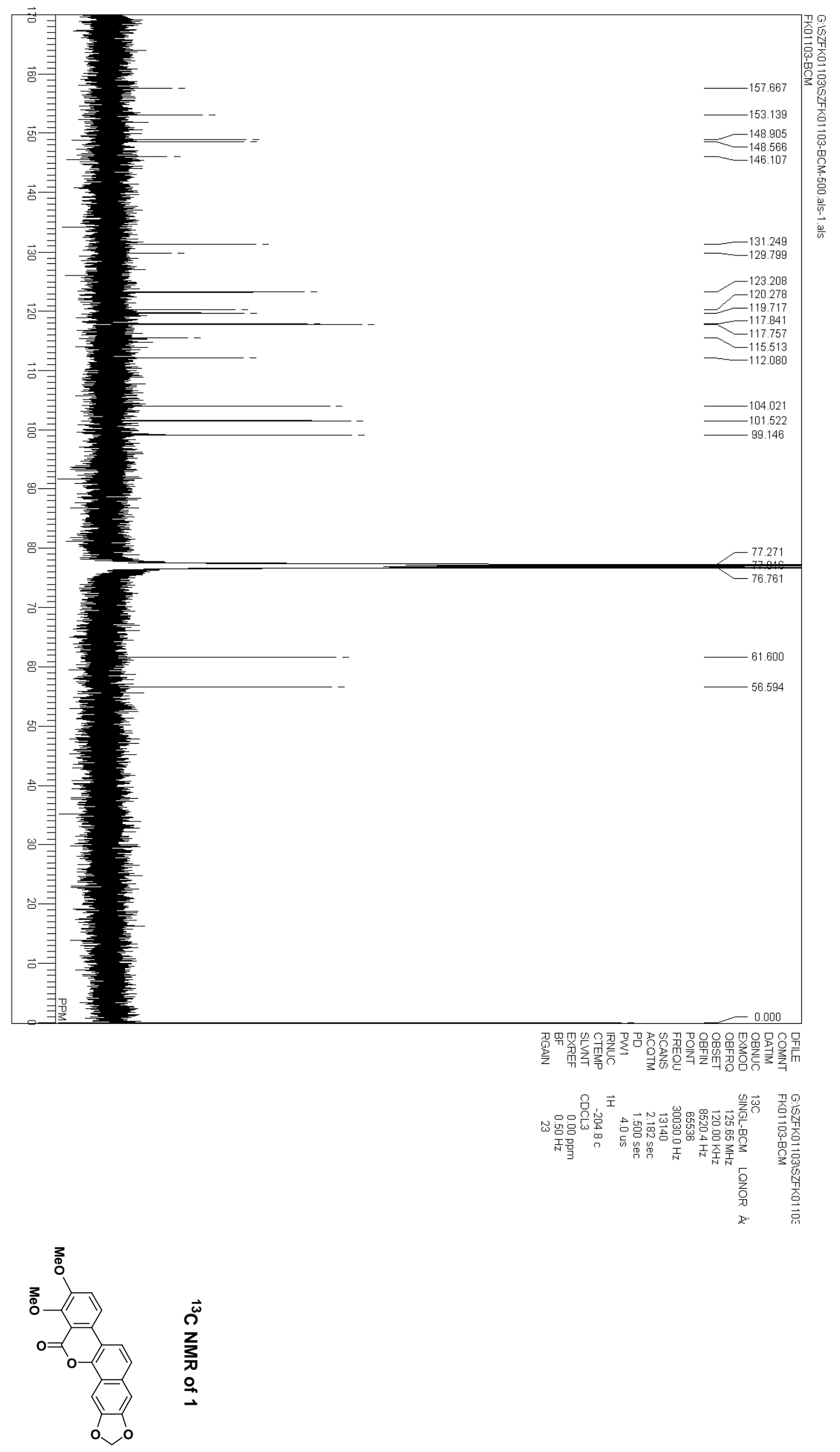



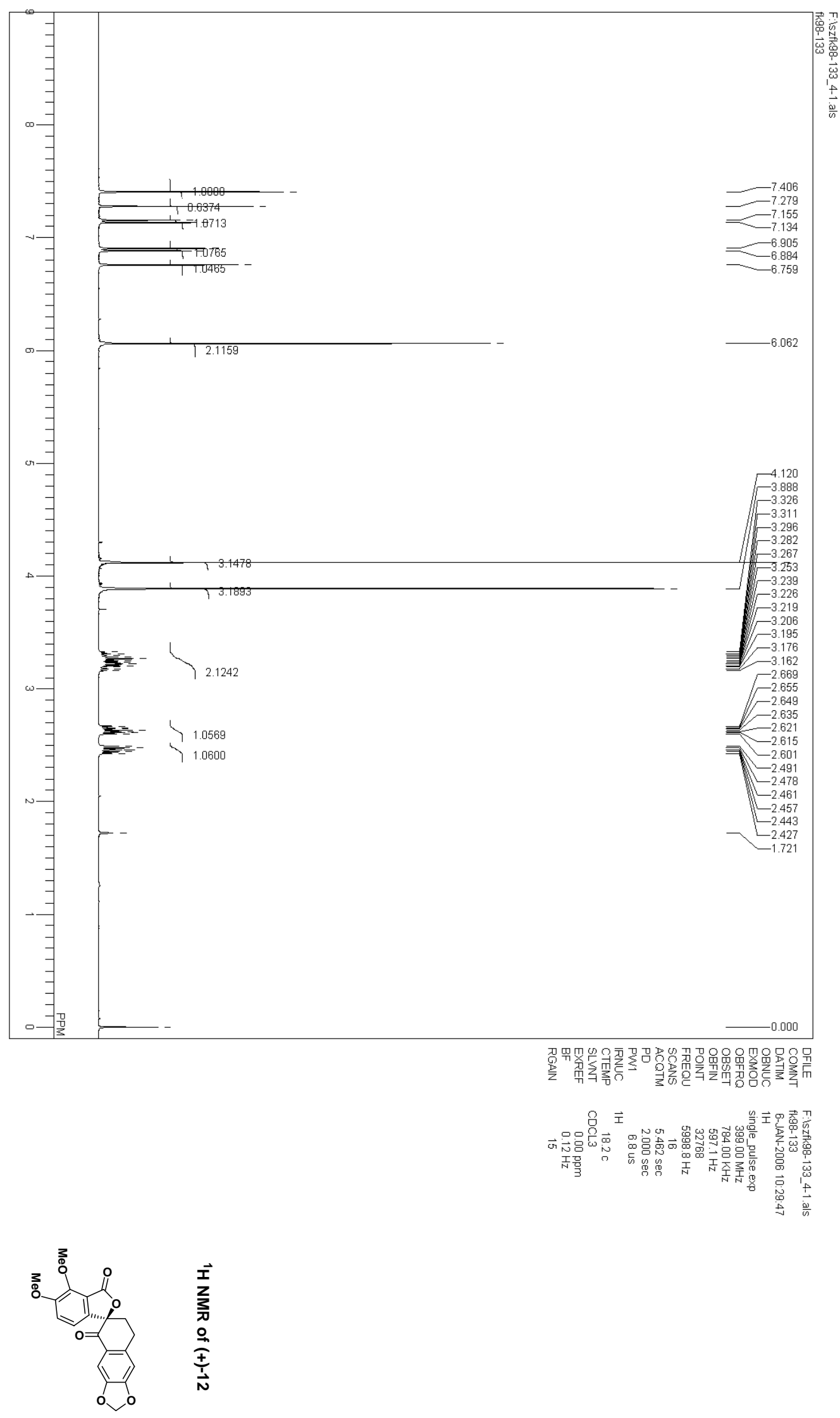

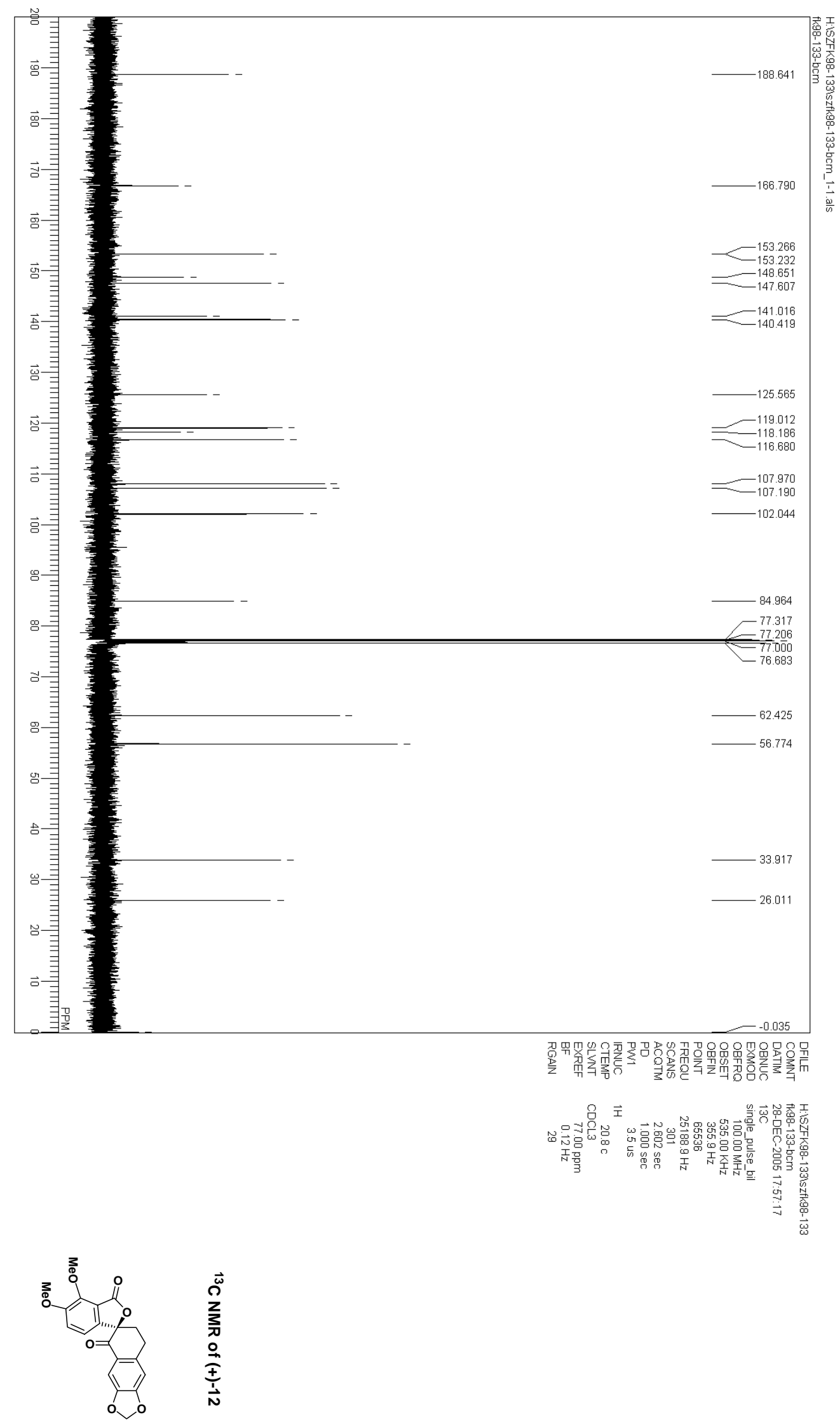
HPLC of $(R)-(+)$-Dihydroarnottin II ((+)-12) (88\% ee)

\section{Chromatogram}

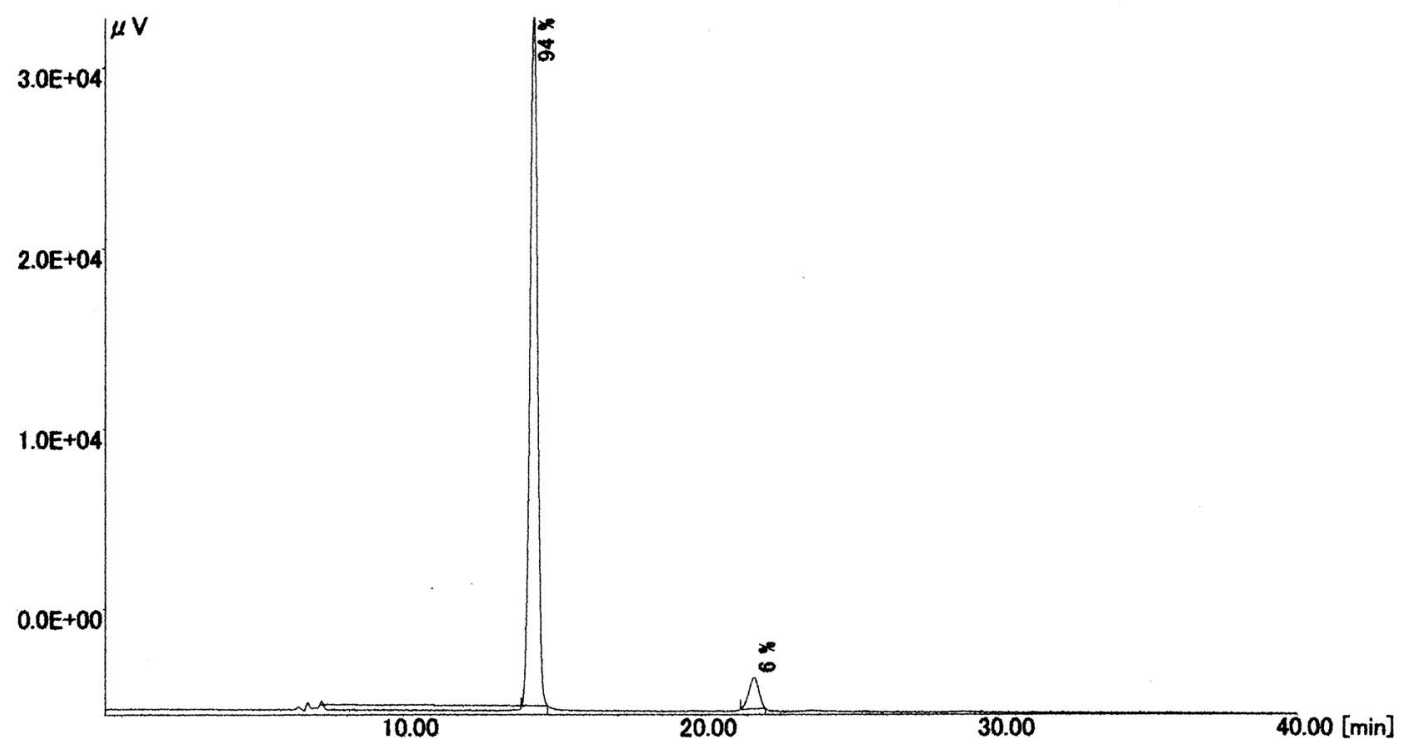

ファイル名: 02001-Si02102. CH3

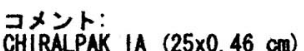

nHexane-CH2CI2: EtOH=15:5:4

$0.5 \mathrm{ml} / \mathrm{min}$

Vial \# = 1 Rack. \# $=1$

注入日：22-Aug-2006 17:01:24

琵在白時: 29-Aug-2006 21:40:44

ユーザー : KONNO

ジテデプログラム:KONNO

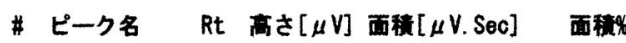

$\begin{array}{rrrrr}1 & 14.375 & 38041 & 648369.962 & 94.17 \\ 2 & 21.792 & 1731 & 40176.250 & 5.83\end{array}$

ピーク

29-Aug-2006 21:40:44,KONNO,Page N-1/1 
HPLC of (S)-(-)-Dihydroarnottin II ((-)-12) (88\% ee)

\section{Chromatogram}

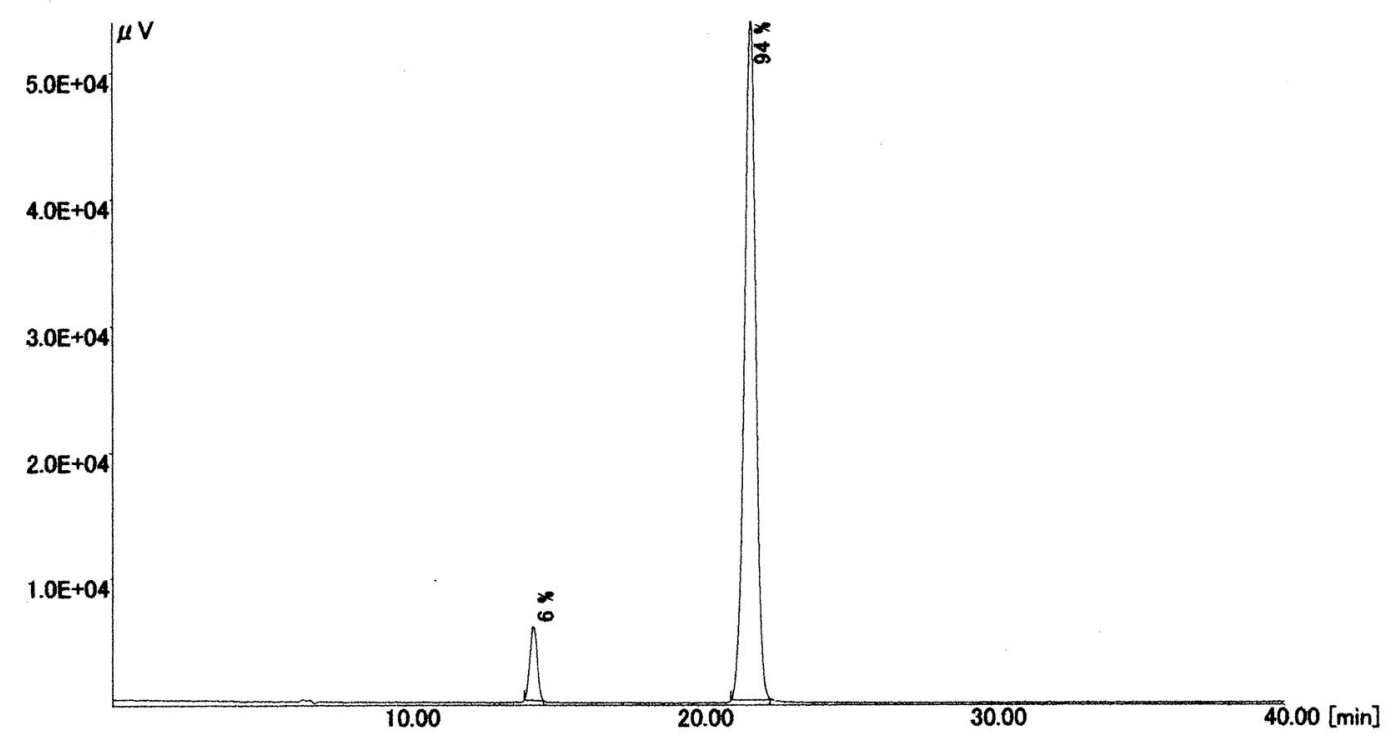

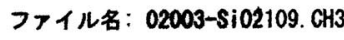

구난

CHIRALPAK IA $(25 \times 0.46 \mathrm{~cm})$

nHexane-CH2CI2: EtOH=15:5:4

$0.5 \mathrm{ml} / \mathrm{min}$.

Vial \# = 1 Rack \# =1

注入星：25-Aug-2006 17:35:38

琵在日時: 25-Aug-2006 18:55:26

ユーザー：KONHO

グループ：FK

システムプログラム:KONNO

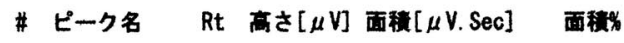

$\begin{array}{rrrrr}1 & 14.350 & 5790 & 95338.800 & 6.35 \\ 2 & 21.758 & 53569 & 1405061.657 & 93.65\end{array}$

ピーク然面楼 $=1500400.457[\mu \mathrm{V} . \mathrm{Sec}]$

25-Aug-2006 18:55:26,KONNO,Page N-1/1 

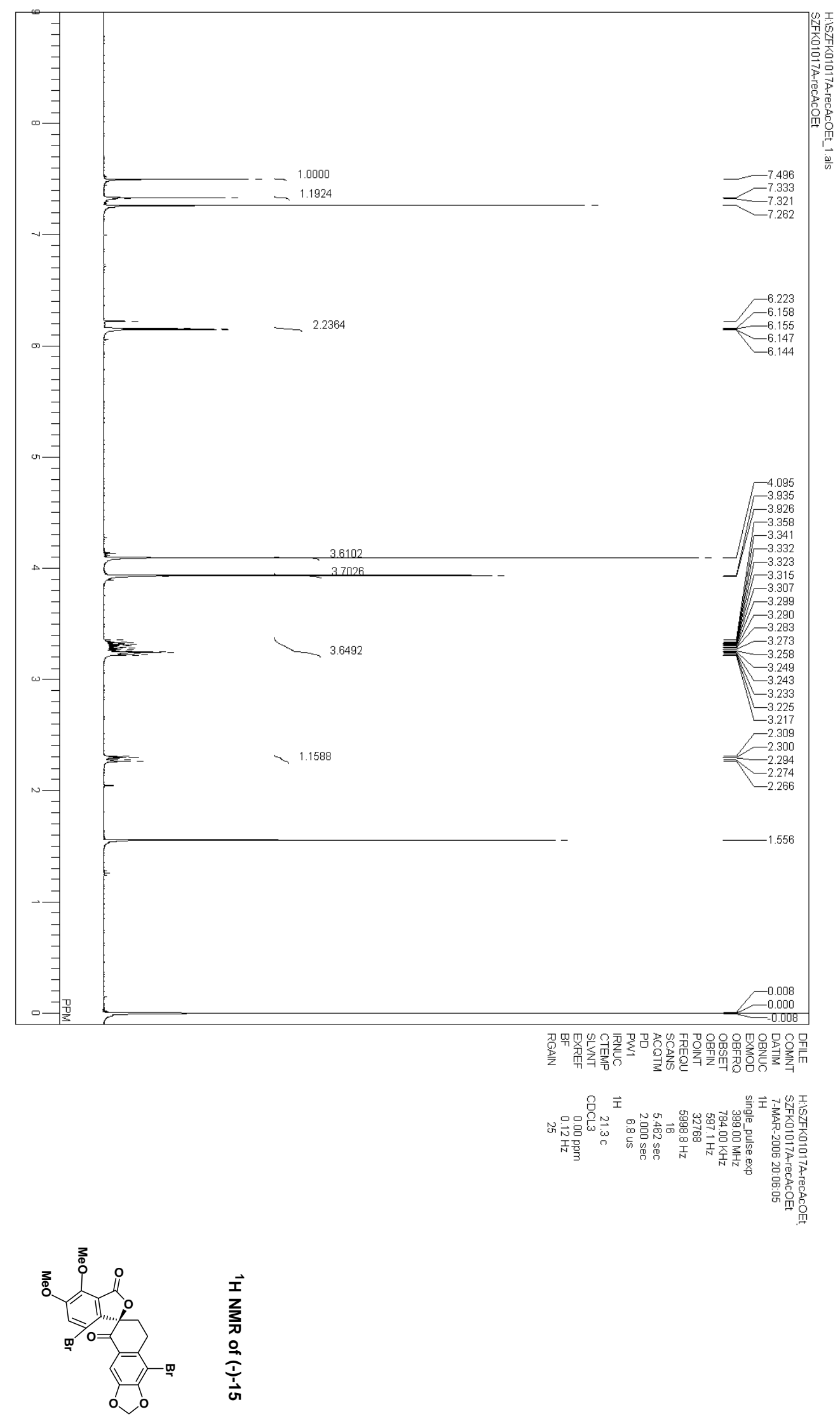

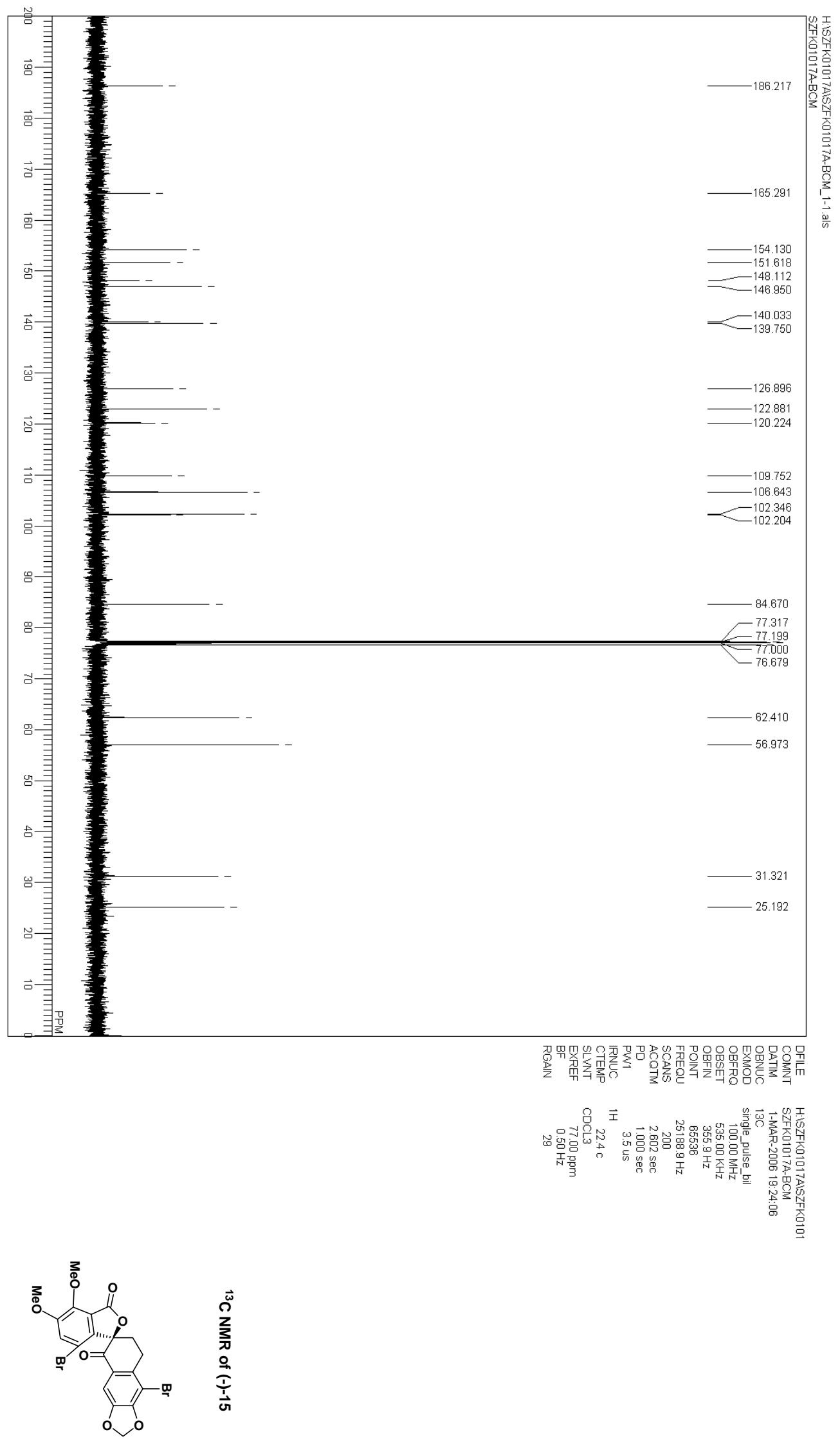
ORTEP Drawing of (R)-(-)-Dibromodihydroarnottin II ((-)-15)

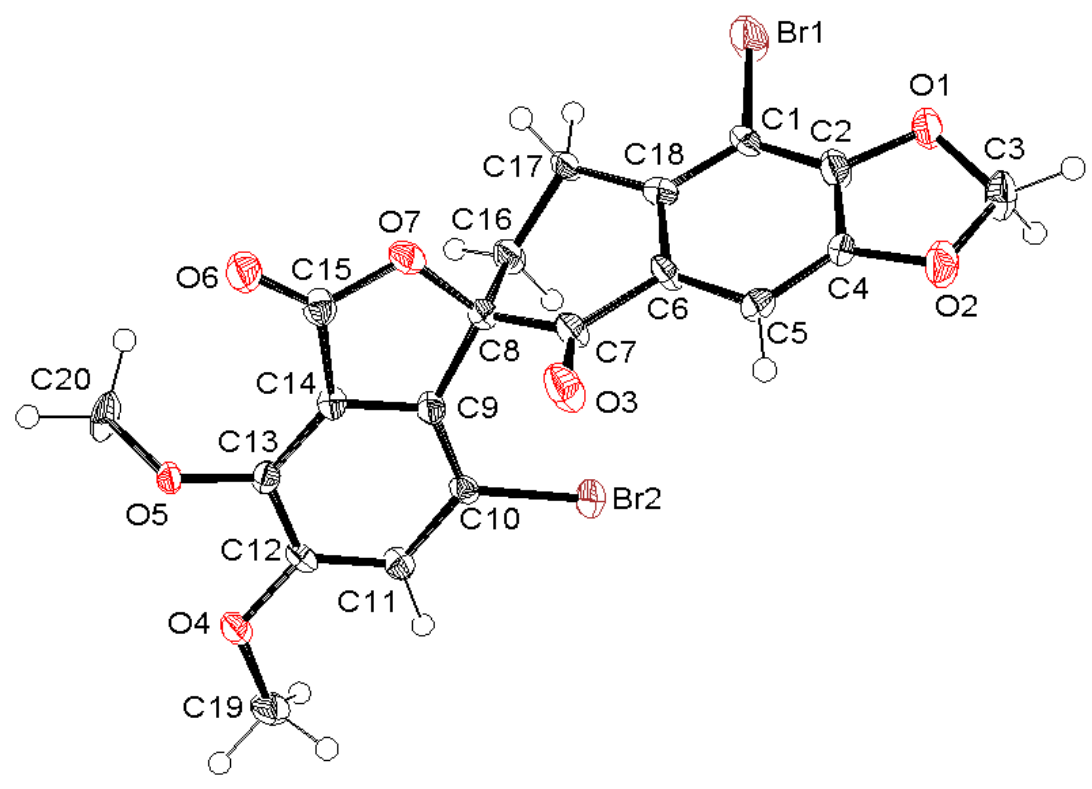




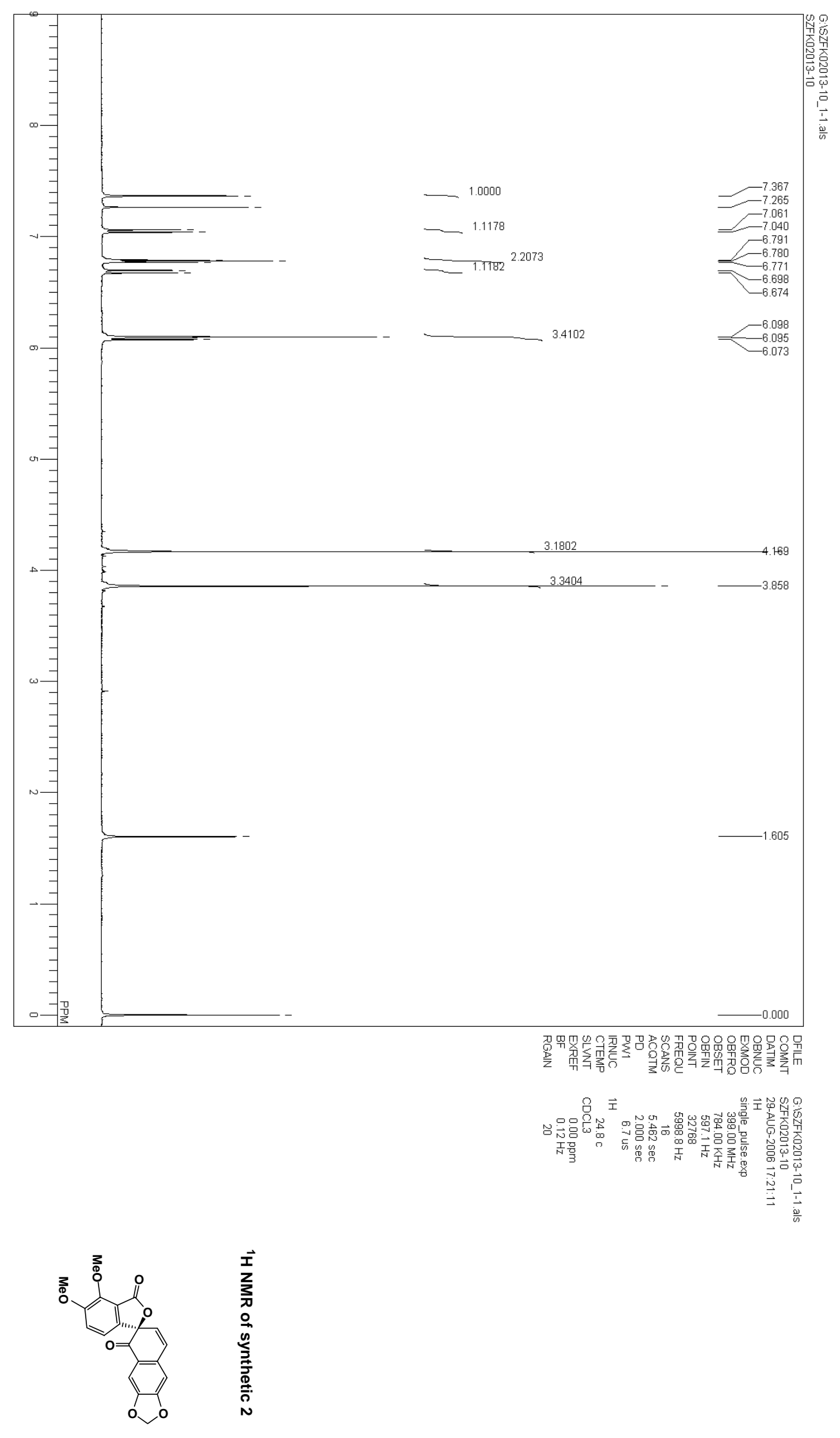



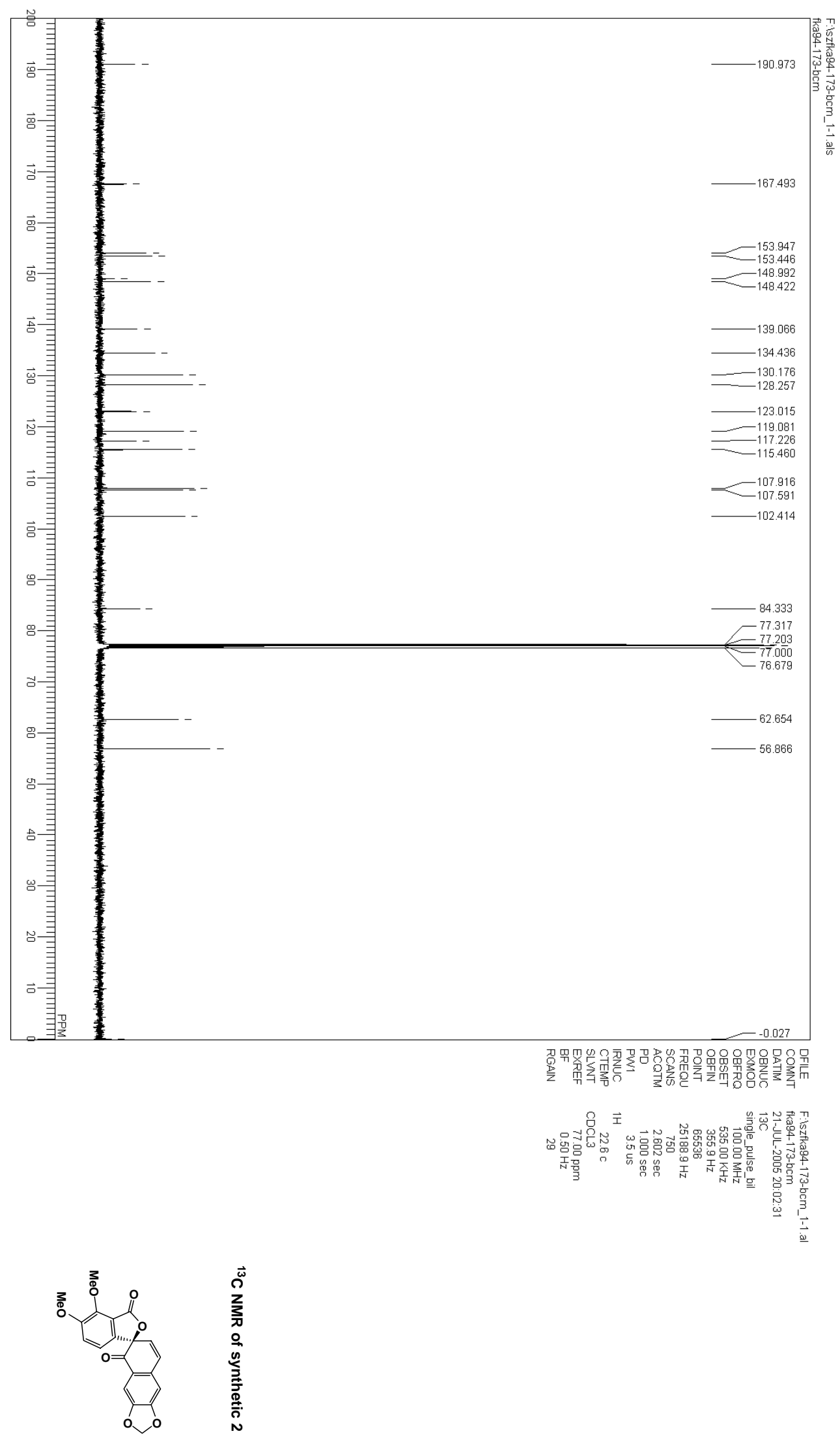
HPLC of (R)-(-)-Arnottin II (2) (synthetic: 98\% ee)

\section{Chromatogram}

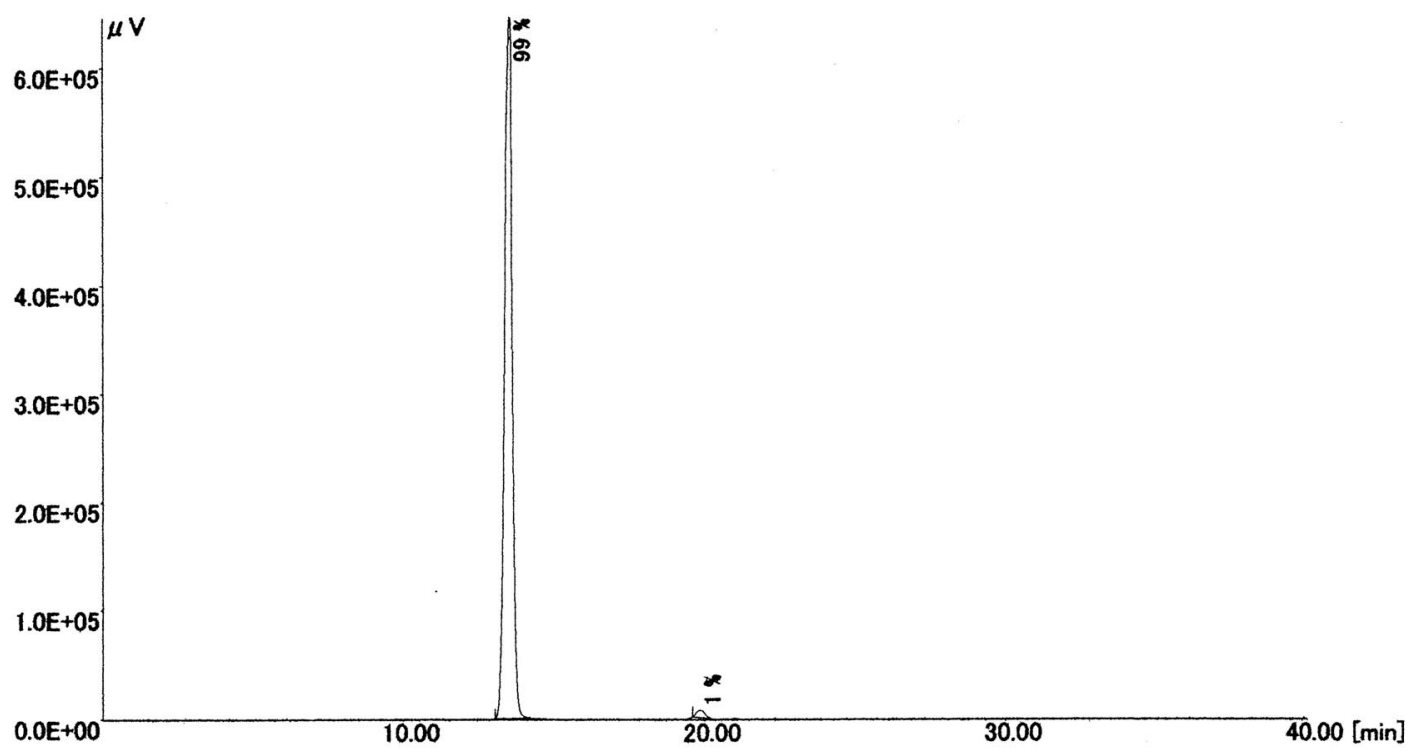

ファイル名：02013-10-rec122. CH3

コメント:

(A $25 \times 0.46 \mathrm{~cm})$

nHexane-CH2CI2: EtOH=15:5:4

$0.5 \mathrm{ml} / \mathrm{min}$.

Vial \#= 1 Rack \# =

注入日：30-Aug-2006 22:26:06

瓄在時: 30 -Aug-2006 23:16:38

フーサニ：KONNO

システムプログラム:KONNO

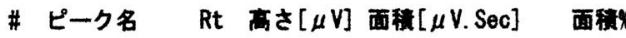

$\begin{array}{rrrrr}1 & 13.500 & 644839 & 10710351.223 & 98.87 \\ 2 & 19.842 & 6718 & 122169.500 & 1.13\end{array}$

ピーク面㮴 $=10832520.723[\mu \mathrm{V} . \mathrm{Sec}]$

30-Aug-2006 23:16:36,KONNO,Page N-1/1 
HPLC of (R)-(-)-Arnottin II (natural: 100\% ee)

\section{Chromatogram}

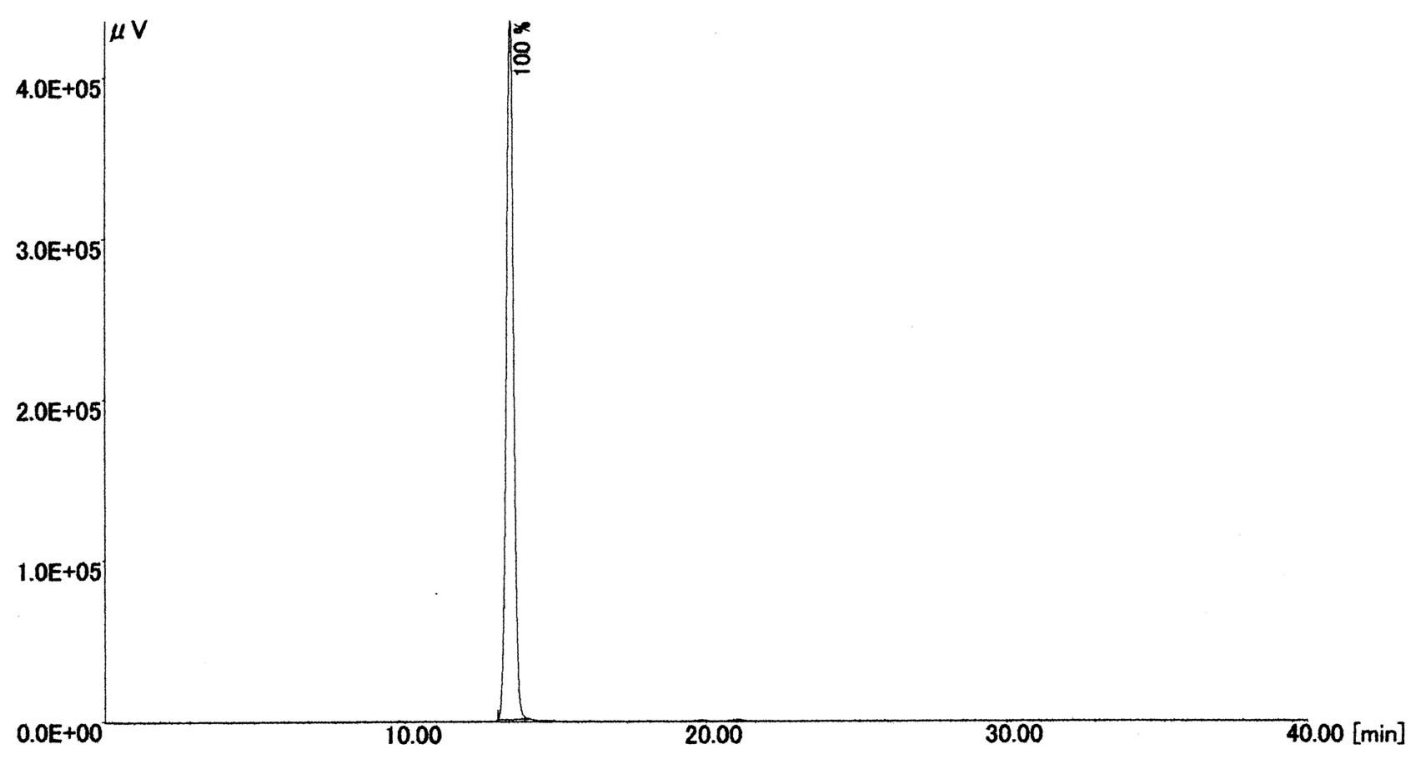

ファイル名: Arnottin II-natural121. CH3

ユコメ̀:

(25)0.46 cm)

nHexane-CH2CI2:EtOHt=15:5:4

$0.5 \mathrm{ml} / \mathrm{min}$.

Vial \# = 1 Rack \# $=1$

注入日: 30-Aug-2006 21:43:08

現在白時: 30 -Aug-2006 22:26:42

ユーザー : KONWO

システムプログラム:KONNO

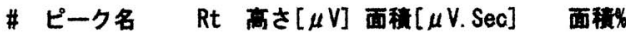

$1 \quad 13.475434139 \quad 6867670.036100 .00$

ピーク絡面皘 $=6867670.036[\mu \mathrm{V} . \mathrm{Sec}]$ 


\section{References}

(1) Kametani, T.; Honda, T.; Inoue, H.; Fukumoto, K. J. Chem. Soc., Perkin Trans. 1 1976, 1221-1225.

(2) Kametani, T.; Honda, T.; Inoue, H. Heterocycles, 1975, 3, 1091-1097.

(3) Marion, N.; Ecarnot, E. C.; Navarro, O.; Amoroso, D.; Bell, A.; Nolan, S. P. J. Org. Chem. 2006, 71, 3816-3821.

(4) Onda, M.; Harigaya, Y.; Horie, J. Heterocycles 1977, 8, 89-95.

(5) Chatterjea, J. N.; Bhakta, S. C.; Chattopadhyay, A. K. J. Indian Chem. Soc. 1974, 51, 757-762.

(6) Ishikawa, T.; Murota, M.; Watanabe, T.; Harayama, T.; Ishii, H. Tetrahedron Lett. 1995, 36, 42694272. 